\title{
EFECTO DE DOSIS DE UREA-N EN EL RENDIMIENTO DEL MAÍZ BAJO UN SISTEMA DE ROTACIÓN CON LEGUMINOSAS DE COBERTURA ${ }^{1}$
}

\author{
H. J. Barreto, C. Pérez, M. R. Fuentes, J. L. Queme, L. Larios ${ }^{2}$
}

\begin{abstract}
RESUMEN
Efecto de dosis de urea-N en el rendimiento del maíz bajo un sistema de rotación con leguminosas de cobertura. La substitución económica de fertilizante nitrogenado por fijación biológica por leguminosas de cobertura en sistemas de rotación con maíz, se encuentra entre los temas de investigación agrícola con mayor potencial para el desarrollo de tecnologías encaminadas a lograr una producción sostenible de maíz en el trópico. En la localidad de Cuyuta, Guatemala, se realizó un experimento durante dos ciclos consecutivos de maíz, para cuantificar la equivalencia entre dosis de urea y $\mathrm{N}$ aportado por dos leguminosas (Canavalia ensiformis L. y Mucuna deeringianum Bort). En el primer ciclo, el rendimiento de maíz respondió significativamente tanto a las aplicaciones de urea-N, como a la incorporación de abono verde de legu-minosas. Durante el segundo ciclo, el efecto residual de las leguminosas en el rendimiento de maíz fue marcadamente menor y las diferencias entre sistemas de rotación dependieron del nivel de $\mathrm{N}$ y la densidad de plantas. El efecto de rotación con abonos verdes y su relación con dosis de urea comprobó la substitución de $\mathrm{N}$ fertilizante por abono verde. Los factores principales que afectaron la respuesta del rendimiento bajo los diferentes sistemas de rotación fueron: dosis de fertilizante $\mathrm{N}$, cantidad de $\mathrm{N}$ en la biomasa superficial de leguminosa $\mathrm{y}$, la densidad final de plantas.
\end{abstract}

\begin{abstract}
Effect of N-urea on corn yield under a rotation system with a leguminous covering. The economical substitution of nitrogenous fertilizer by biological fixation by a leguminous covering under rotation systems with corn (Zea mays L.), is one of the agricultural research themeswith highest potential for developing technologies aimed to achieve a sustainable corn production in the tropics. An experiment was conducted during twoconsecutive corn cycles at Cuyuta-Guatemala, in order to quantify the equivalence of urea dos es and the $\mathrm{N}$ supplied by two legumes (Canavalia ensiformis L. and Mucuna deeringianum Bort). During the first cycle, the corn yield showed a significant response to the $\mathrm{N}$-urea as well as to the incorporation of green manure. During the second cycle, the residual effect of the legumes on the corn yield was markedly lower and the differences among rotation systems depended on the $\mathrm{N}$ level and plant population. The rotation effect with green manure and its relation with the doses of urea approved the substitution of $\mathrm{N}$-fertilizer by green manure. The main factors affecting the yield response under the different rotation systems were: doses of $\mathrm{N}$-fertilizer, amount of $\mathrm{N}$ in the superficial biomass of the legume and the final plant population.
\end{abstract}

\section{INTRODUCCIÓN}

En Centro América y El Caribe se siembran anualmente dos millones de hectáreas de maíz en las que la producción económica demanda cantidades considerables de insumos externos, particularmente de fertilizante nitrogenado, debido a que la capacidad de los suelos tropicales bajo cultivo intensivo es en general insuficiente para suplir las cantidades necesarias del elemento de acuerdo a las necesidades del cultivo. La fertilización del maíz en estos sistemas varía ampliamente, pero las fuentes predominantes son el sulfato de amonio y la urea (Armuelles, 1992). La National Academy of Sciences (1991) sugiere que los sistemas sostenibles de producción de cultivos deben incluir el reciclaje de nutrientes y la aplicación racional de la labranza, como elementos esenciales de la estrategia de

1 Presentado en la XXXIX Reunión Anual del PCCMCA en Guatemala, América Central. 28 de marzo - 3 de abril, 1993.

2 Contribución conjunta del CIMMYT (Guat.). Programa de Maíz del Instituto de Ciencia y Tecnología Agropecuaria (ICTA), Guatemala. 
conservación para mantener la productividad de los sistemas agrícolas, con mínimo impacto negativo sobre el recurso base. La literatura para producción de granos en el trópico húmedo aconseja la incorporación de leguminosas de cobertura para la fijación biológica de $\mathrm{N}$ disminuyendo así la necesidad de aplicar fertilizantes químicos (Wade y Sanchez, 1983; Yost et al., 1985). Sin embargo, la selección de leguminosas apropiadas, las posibilidades de inserción a los diferentes sistemas de producción, y la evaluación de la factibilidad económica de la substitución de fertilizante $\mathrm{N}$, aún requieren de mayor evaluación, antes de obtener recomendaciones confiables para la producción comercial de maíz en rotación con leguminosas de cobertura en el trópico bajo de Centro América.

Smyth et al. (1991) indican valores de substitución de $\mathrm{N}$ fijado en forma biológica de 26 y $74 \mathrm{~kg} \mathrm{~N} / \mathrm{ha}$ de $\mathrm{N}$ fertilizante en sistemas de rotación en suelos tropicales utilizando Vigna sp. y Mисиna sp. Sin embargo, se ha demostrado experimentalmente que es posible obtener valores de substitución de $\mathrm{N}$ de hasta $170 \mathrm{~kg} \mathrm{~N} /$ ha utilizando otras leguminosas de cobertura (Bouldin et al., 1989). Como parte de un proyecto de investigación sobre sistemas de rotación de maíz con leguminosas de cobertura en el trópico bajo de Guatemala, se realizó un ensayo con los siguientes objetivos:
1. Cuantificar la equivalencia entre el $\mathrm{N}$ proveniente de urea y el $\mathrm{N}$ aportado por Canavalia ensiformis L. y Mucuna deeringianum Bort. a través de dos ciclos consecutivos de producción de maíz.

2. Evaluar el efecto de los factores genotipo y tratamiento de semilla con insecticida en relación a la respuesta a la fertilización con $\mathrm{N}$ bajo diferentes sistemas de rotación.

3. Determinar la importancia agronómica de las interacciones entre los factores bajo estudio y sus implicaciones para la utilización de rotaciones con leguminosas de cobertura para la producción de maíz.

\section{MATERIALES Y MÉTODOS}

Este experimento fue realizado en la estación experimental Cuyuta localizada al sureste de Guatemala a 30 msnm. El área experimental está constituida por una planicie aluvial que ha recibido aportes de materiales viroclásticos (e.g. ceniza volcánica) en diferentes períodos de tiempo. La suelo tiene textura franco arenosa, $\mathrm{pH} 6,5$, bajos contenidos disponibles de $\mathrm{N}$ y $\mathrm{P}$ y moderados de $\mathrm{K}$. Durante el año hay una estación principal de cultivo entre junioseptiembre. Sin embargo, con irrigación es factible obtener un segundo ciclo entre diciembre y marzo. La secuencia de rotaciones y sistemas de labranza para el experimento se presentan en el Cuadro 1.

Cuadro 1. Sistemas de rotación evaluados para la producción de maíz, Cuyuta Guatemala.

\begin{tabular}{llll}
\hline Rotación & \multicolumn{3}{c}{ Sistemas de rotación y labranza 1991-1992. } \\
\cline { 2 - 4 } & \multicolumn{1}{c}{ 1991A } & \multicolumn{1}{c}{ 1991B } & \multicolumn{1}{c}{ 1992A } \\
\hline 1 & Mucuna & Maíz & Maíz \\
& Labranza Cero & Labranza Convencional & Labranza Cero \\
& Canavalia & Maíz & Maíz \\
& Labranza Cero. & Labranza Convencional & Labranza Cero \\
& Descanso & Maíz & Maíz \\
\hline & Labranza Cero & Labranza Convencional & Labranza Cero
\end{tabular}


El experimento se estableció en junio 1991 en un área homogénea de aproximadamente 0,5 ha. El lote se dividió en tres fajas de aproximadamente $1500 \mathrm{~m}^{2}$ cada una. El primer lote fue dejado en descanso, el segundo se sembró con Canavalia ensiformis L. a $50 \mathrm{~kg}$. semilla/ha, y el tercero con Mucuna deeringianum Bort. a $25 \mathrm{~kg}$ semilla/ha. En diciembre 1991, el suelo en el área experimental fue preparado bajo labranza convencional para irrigación por surcos durante el ciclo de maíz. El término labranza convencional se refiere a la preparación de suelo utilizando discos y rastra para lograr la incorporación completa del material superficial. Previo a la incorporación del residuo superficial se cuantificó la producción de biomasa de cada leguminosa y se tomaron muestras de suelo en cada lote de rotación (Cuadro 2).

En diciembre 1991, en cada lote de rotación se superimpuso un experimento con idénticos tratamientos. El experimento considera. tres dosis de nitrógeno $(0,75 \mathrm{y}$ $150 \mathrm{Kg}$. N/ha como urea), tratamiento de semilla con insecticida (i.a. 2,3 dihidro 2,2, dimetil 7 Benzofuran [(dibutilamina)tio] metil carbamato]) antes de la siembra (S0 $=\sin$ y $\mathrm{S} 1=\mathrm{con}$ ), y dos genotipos de maíz ( V0=B1 y $\mathrm{Vl}=\mathrm{HB} 85)$. El arreglo de tratamientos fue de factorial $3 \times 2^{2}$ balanceado.
El diseño experimental utilizado fue de bloques incompletos con tres repeticiones por tratamiento. En este diseño la interacción $\mathrm{SxV}$ se encuentra parcialmente confundida con el efecto de bloques. La interacción $\mathrm{NxSxV}$ se encuentra completamente confundida con el efecto de bloques dentro de cada repetición. En junio 1992, se repitió el experimento con la misma aleatorización en cada lote de rotación pero el manejo fue bajo labranza cero. Labranza cero se refiere a la ausencia de operaciones mecánicas al suelo y al manejo superficial del residuo distribuido uniformemente sobre el terreno.

Las 108 parcelas recibieron un manejo uniforme con respecto .al control de malezas con herbicida preemergente, dosis de fertilización con fósforo $\left(40 \mathrm{Kg} / \mathrm{ha} \mathrm{P}_{2} \mathrm{O}_{5}\right.$ como superfosfato triple), y control de insectos del follaje. En los tratamientos que recibieron $\mathrm{N}$, se aplico $50 \%$ a la siembra junto con el P. El 50\% restante se aplicó 30 días después de la siembra del maíz.

Las unidades experimentales consistieron de seis surcos de maíz de $6 \mathrm{~m}$ de longitud $\left(35 \mathrm{~m}^{2}\right)$. El tamaño del bloque dentro de cada repetición fue de $3 \times 2$ unidades experimentales $\left(210 \mathrm{~m}^{2}\right)$. A la cosecha se determinaron el rendimiento de maíz, contenido de humedad de grano, número de plantas y número de mazorcas sanas y podridas.

Cuadro 2. Análisis de suelo en campos con y sin leguminosa antes de la siembra de los experimentos con maíz. Cuyuta, Guatemala. 1991.

\begin{tabular}{lcccc}
\hline & \multicolumn{3}{c}{ SISTEMA DE ROTACION } \\
\cline { 2 - 5 } Análisis & Sin leguminosa & Canavalia & Mucuna & Promedio \\
\hline $\mathrm{pH} 1: 2,5$ & 6,5 & 6,5 & 6,7 & 6,6 \\
MO \% & 3,3 & 4,0 & 3,4 & 3,6 \\
$\mathrm{CIC}$ meq/100 & 11,9 & 8,7 & 10,0 & 10,2 \\
NH4-N mg/kg & 53 & 49 & 44 & 49 \\
NO3-N mg/kg & 6,5 & 8,1 & 12,6 & 9,1 \\
P mg/kg & 9,5 & 10,9 & 18,9 & 13,1 \\
K meq/100g & 1,0 & 0,7 & 0,7 & 0,8 \\
Ca meq/100g & 8,8 & 6,1 & 6,9 & 7,3 \\
Mg meq/100g & 1,4 & 1,2 & 1,8 & 1,5 \\
Na meq/100g & 0,4 & 0,4 & 0,4 & 0,4 \\
\hline
\end{tabular}


Todas las operaciones de siembra y cosecha se realizaron en forma manual.

Se realizó un análisis de varianza combinado a través de los sistemas de rotación. Para las comparaciones preplaneadas se realizaron contrastes noortogonales de un grado de libertad.

\section{RESULTADOS Y DISCUSIÓN}

\section{Producción de Biomasa por las Leguminosas}

La amplia adaptabilidad de Canavalia ensiformis L. y Mucuna deeringianum a diversas condiciones climáticas en sistemas de producción de maíz de la región Centroamericana han sido reportadas por Zea et al., (1991). El desarrollo de las leguminosas durante el ciclo de temporal 1991A fue uniforme y abundante, confirmando la adaptación de estas a las condiciones climáticas y de suelo del área experimental. Los muestreos realizados indicaron que Canavalia ensiformis L. produjo 10,8 t/ha de biomasa seca mientras que Mucuna deeringianum produ- jo 7,5 t/ha. El Cuadro 3 presenta el análisis químico elemental ( $\mathrm{C}, \mathrm{N}, \mathrm{P}$ y $\mathrm{K}$ ) de las muestras de biomasa para las dos leguminosas. El total de nutrientes contenido en la biomasa superficial de las leguminosas se presenta en el Cuadro 4. Es evidente que los contenidos de nutrientes aportados por las leguminosas son de importancia agronómica, al menos en términos de contenidos absolutos de N y K. Los análisis de suelo de los lotes en rotación, previo a la preparación del terreno y siembra de los ensayos con maíz, no presentaron mayores diferencias en su caracterización química, aunque se noto una ligera tendencia del $\mathrm{NO}_{3}-\mathrm{N}$ y el $\mathrm{P}$ a aumentar en los lotes con leguminosas (Cuadro 2).

\section{Respuesta del Sistema de Rotación a Dosis de Urea}

El análisis de varianza combinado a través de sistemas de rotación para los dos ciclos de evaluación se presenta en el Cuadro 5. Los promedios para rendimiento de grano en relación a la aplicación de $\mathrm{N}$ para las diferentes combinaciones de tratamientos, durante los dos ciclos de evaluación se presentan en el Cuadro 6.

Cuadro 3. Análisis químico elemental de biomasa superficial de dos leguminosas de cobertura. Cuyuta Guatemala.

\begin{tabular}{lccccc}
\hline \multicolumn{5}{c}{ Elemento } \\
\hline Leguminosa & $\mathrm{C} \%$ & $\mathrm{~N} \%$ & $\mathrm{C} / \mathrm{N}$ & $\mathrm{P} \%$ & $\mathrm{~K} \%$ \\
Mucuna sp. & 34,33 & 2,155 & 15,9 & 0,145 & 0,650 \\
Canavalia ensiformis & 34,58 & 2,280 & 15,2 & 0,190 & 0,970 \\
\hline
\end{tabular}

Cuadro 4. Aporte de nutrientes en la biomasa superficial de dos leguminosas de cobertura. Cuyuta Guatemala.

\begin{tabular}{lccccc}
\hline & \multicolumn{5}{c}{ Contenido de nutrientes } \\
\cline { 2 - 6 } & Biomasa & $\mathbf{C}$ & N & P & K \\
\hline Leguminosa & & & $\mathrm{kg} / \mathrm{ha}$ & \\
Mucuna sp. & 7500 & 2585 & 162 & 11 & 49 \\
Canavalia ensiformis & 10800 & 5629 & 246 & 21 & 105 \\
\hline
\end{tabular}


Cuadro 5. Análisis de varianza combinado a través de sistemas de rotación para rendimiento de grano de maíz durante dos ciclos de evaluación. Cuyuta Guatemala.

\begin{tabular}{|c|c|c|c|c|c|}
\hline \multirow[b]{3}{*}{ FUENTE } & \multirow[b]{3}{*}{ G.L. } & \multicolumn{3}{|c|}{ Análisis de variancia } & \\
\hline & & \multicolumn{2}{|c|}{ 1991B } & \multicolumn{2}{|c|}{$1992 A$} \\
\hline & & Cuad.Med & Signif. & Cuad.Med & Signif. \\
\hline ROT & 2 & 39,44 & $* *$ & 1,24 & $*$ \\
\hline ROT(REP) & 14 & 0,15 & & 0,06 & \\
\hline $\mathrm{N}$ & 2 & 19,48 & $* *$ & 14,26 & $* *$ \\
\hline S & 1 & 27,44 & $* *$ & 87,03 & $* *$ \\
\hline V & 1 & 8,20 & $* *$ & 0,69 & \\
\hline $\mathrm{NxS}$ & 2 & 0,83 & & 4,19 & $* *$ \\
\hline $\mathrm{NxV}$ & 2 & 0,12 & & 0,29 & \\
\hline $\mathrm{SxV}$ & 1 & 3,34 & $*$ & 7,65 & $* *$ \\
\hline $\mathrm{NxSxV}$ & 2 & 0,76 & & 0,61 & \\
\hline ROTxN & 4 & 7,79 & $* *$ & 1,24 & $* *$ \\
\hline ROTxS & 2 & 0,78 & & 4,41 & $* *$ \\
\hline ROTxV & 2 & 1,91 & $*$ & 0,11 & \\
\hline ROTxNxS & 4 & 0,14 & & 0,41 & \\
\hline ROTxNxV & 4 & 1,23 & @ & 0,27 & \\
\hline ROTxSxV & 2 & 3,68 & $* *$ & 0,38 & \\
\hline ROTxNxSxV & 4 & 0,77 & & 0,03 & \\
\hline ERROR & 66 & 0,61 & & 0,36 & \\
\hline TOTAL & 107 & & & & \\
\hline
\end{tabular}

Los símbolos @ , * ,**indican significancia estadística al nivel de probabilidad de 10,5 y $1 \%$, respectivamente.

Durante el ciclo 1991B, las diferencias en rendimiento entre sistemas de rotación, fueron estadísticamente significativas para la comparación entre el sistema en descanso $(4,2 \mathrm{t} / \mathrm{ha})$ y el efecto simple de rotación con cualquiera de las dos leguminosas (6,0 t/ha). En el sistema de rotación sin leguminosa, el $\mathrm{N}$ mostró un comportamiento lineal con ganancias significativas de rendimiento de grano de 1,95 y $1,15 \mathrm{t} / \mathrm{ha}$, respectivamente para dosis de 75 y $150 \mathrm{Kg}$. N/ha a través de genotipos. En el lote con rotación de Mucuna deeringianum, el rendimiento de gra- no también respondió linealmente a dosis de urea- $\mathrm{N}$, aunque en menor magnitud y no significativo para el incremento de 75 a $150 \mathrm{Kg}$. N/ha. Bajo el sistema de rotación con Canavalia ensiformis L. la respuesta a dosis de $\mathrm{N}$ no fue significativa a $\mathrm{P}<0,05$.

Durante el ciclo 1992A, el rendimiento promedio de los sistemas de rotación disminuyó considerablemente. La razones de está drástica disminución del rendimiento en los diferentes sistemas de rotación no están claramen- 
Cuadro 6. Promedios de rendimiento de grano de maíz a $15 \%$ humedad (t/ha) para niveles de urea- $\mathrm{N}(\mathrm{kg} / \mathrm{ha})$, contenido de $\mathrm{N}$ en biomasa superficial de abono verde $(\mathrm{kg} / \mathrm{ha})$ y combinaciones de tratamiento de semilla $(\mathrm{S})$ genotipo (V) para dos ciclos de evaluación (91B y 92A), Cuyuta, Guatemala.

\begin{tabular}{lccccccccc} 
urea-N & leg & S0V091B & S0V191B & S1V091B & S1V191B & S0V092A & S0V192A & S1V092A & S1V192A \\
\hline 0 & 0 & 1,98 & 2,46 & 3,26 & 2,38 & 0,9 & 0,83 & 1,27 & 1,1 \\
75 & 0 & 3,66 & 3,85 & 4,66 & 5,74 & 1,94 & 1,76 & 3,2 & 4,09 \\
150 & 0 & 4,3 & 6,11 & 5,86 & 6,24 & 2,46 & 1,93 & 3,03 & 3,31 \\
0 & 246 & 5,36 & 5,32 & 5,74 & 7,29 & 0,78 & 0,45 & 1,74 & 2,44 \\
75 & 246 & 6,24 & 5,2 & 6,72 & 7,62 & 1,05 & 0,42 & 2,9 & 4,15 \\
150 & 246 & 5,91 & 4,45 & 6,13 & 6,9 & 0,89 & 0,52 & 3,24 & 4,08 \\
0 & 162 & 5,04 & 4,87 & 4,81 & 6,42 & 0,52 & 0,83 & 1,41 & 2,37 \\
75 & 162 & 5,19 & 6 & 5,82 & 7,71 & 1,18 & 0,52 & 2,35 & 3,43 \\
150 & 162 & 5,62 & 6,93 & 6,19 & 7,02 & 1,38 & 0,49 & 3,32 & 3,72 \\
\hline
\end{tabular}

te establecidas; sin embargo, los factores climáticos, el manejo bajo labranza cero, y una menor densidad de siembra durante este ciclo, posiblemente afectaron la respuesta a los factores bajo estudio.

\section{Substitución de Fertilizante N}

La tendencias del rendimiento para el ciclo 1991B indican que el efecto de rotación es de alta importancia agronómica y que además existe una fuerte interacción con dosis de $\mathrm{N}$, lo que confirma el potencial de substitución entre las dos fuentes. La evidencia de interacción rotación x $\mathrm{N}$ como tal, no es suficiente para cuantificar la equivalencia entre el $\mathrm{N}$ proveniente de urea y el aportado por las leguminosas al incorporarlas al suelo, dado que es difícil establecer con exactitud la cantidad de $\mathrm{N}$ disponible aportado por cada leguminosa durante la descomposición de los residuos. Sin embargo, el total de N en la biomasa superficial de Canavalia ensiformis L. fue aproximadamente $66 \%$ superior a la cantidad aportada por Mucuna deeringianum, ambos en proporción directa a la cantidad de biomasa superficial producida (Cuadro 4). Asimismo, la baja respuesta a dosis de $\mathrm{N}$ en sistemas de rotación con leguminosas, sugiere que la cantidad de $\mathrm{N}$ aportada fue suficiente para producir altos rendimientos, aún en la ausencia de fertilización con N. Sin embargo, se debe anotar que en el sistema de rotación con Canavalia ensiformis L. se observó una tendencia del rendimien- to a disminuir a dosis de $150 \mathrm{Kg}$. N/ha (Cuadro 6).

El contenido de $\mathrm{N}$ en la biomasa superficial de las leguminosas permite obtener una aproximación de la cantidad del elemento potencialmente disponible para reciclaje lo que ofrece un elemento cuantitativo para la relación con la respuesta a dosis de $\mathrm{N}$ en presencia o ausencia de abono verde. Sin embargo, la selección del modelo apropiado para expresar esta relación en forma cuantitativa y la asignación de relaciones de precios apropiadas para establecer la tasas de substitución económica, se encuentran todavía en proceso de evaluación y por lo tanto no se discutirán en este trabajo.

\section{Efecto del Tratamiento de Semilla en la Respuesta a Dosis de Urea}

El tratamiento de la semilla de maíz con insecticida al momento de la siembra fue el efecto experimental más consistente en los diferentes sistemas evaluados. El impacto del uso de insecticida como tratamiento de semilla se manifestó claramente en el componente número de plantas, el cual fue determinante para modificar el número de mazorcas totales y por lo tanto el rendimiento de grano en los diferentes tratamientos (Cuadro 7). Durante el ciclo 1991B, la disminución promedio de plantas en los tratamientos sin insecticida fue alrededor de $12.000 \mathrm{pl} / \mathrm{ha}$, lo que fue equivalente a $1 \mathrm{t} / \mathrm{ha}$ de grano. Durante 1992A, 
Cuadro 7. Promedios de número de plantas por $\mathrm{m}^{2}$ para niveles de urea- $\mathrm{N}$, contenido de $\mathrm{N}$ en biomasa superficial de abono verde y combinaciones de tratamiento de semilla (S) genotipo (V) para dos ciclos de evaluación (91B y $92 \mathrm{~A})$, Cuyuta, Guatemala.

\begin{tabular}{lccccccccc}
\hline urea-N & N leg & S0V091B & S0V191B & S1V091B & S1V191B & S0V092A & S0V192A & S1V092A & S1V192A \\
\hline 0 & 0 & 4,99 & 2,62 & 4,94 & 4,49 & 1,73 & 1,34 & 2,56 & 2,18 \\
75 & 0 & 4,43 & 2,28 & 5,23 & 5,00 & 1,92 & 1,31 & 3,06 & 3,01 \\
150 & 0 & 4,94 & 4,02 & 5,81 & 5,28 & 2,16 & 1,31 & 2,48 & 2,56 \\
0 & 246 & 4,91 & 3,55 & 5,16 & 5,31 & 1,21 & 0,50 & 2,68 & 2,82 \\
75 & 246 & 4,87 & 3,05 & 5,47 & 5,36 & 1,07 & 0,37 & 2,58 & 2,85 \\
150 & 246 & 5,07 & 2,51 & 4,89 & 4,70 & 0,71 & 0,49 & 2,74 & 2,74 \\
0 & 162 & 4,59 & 3,52 & 5,23 & 5,13 & 0,88 & 1,08 & 2,73 & 2,89 \\
75 & 162 & 4,34 & 3,73 & 5,40 & 5,06 & 1,08 & 0,54 & 2,39 & 2,88 \\
150 & 162 & 4,73 & 4,49 & 5,65 & 5,04 & 1,14 & 0,43 & 2,78 & 2,66 \\
\hline
\end{tabular}

la reducción promedio de población de plantas en los tratamientos sin insecticida a través de sistemas de rotación fue de alrededor de $16.000 \mathrm{pl} / \mathrm{ha}$, lo que ocasionó una disminución del rendimiento de aproximadamente $1,8 \mathrm{t} / \mathrm{ha}$. Cabe anotar que durante este ciclo se observó un efecto altamente significativo de interacción de tratamiento de semilla por nivel de rotación, resultando en rendimientos muy bajos ( $<1 \mathrm{t} / \mathrm{ha})$ en los sistemas que tuvieron leguminosa de cobertura en 1991A. Estos resultados sugieren que la población de insectos de suelo durante el segundo ciclo de maíz fue mayor en los tratamientos que tuvieron leguminosa de cobertura; sin embargo, la naturaleza de este efecto no esta establecida y requiere de una evaluación posterior.

La respuesta a dosis de $\mathrm{N}$ fue claramente afectada por la población de plantas, particularmente durante el segundo ciclo de maíz. Al tomar la población de plantas como indicativo, se observó una disminución de la respuesta a dosis de $\mathrm{N}$ en función de la densidad, notándose una ausencia de respuesta cuando las poblaciones de plantas estuvieron por debajo de $1,2 \mathrm{pl} / \mathrm{m}^{2}$. Los resultados de este estudio sugieren que la protección de la semilla y su relación con el establecimiento de las densidades críticas de plantas, deben ser consideradas en forma integrada para entender la variabilidad de respuesta cuando se evalúan sistemas de rotación con leguminosas de cobertura.

\section{Efecto del Genotipo en la Respuesta a Dosis de urea}

La ausencia de interacción de genotipo por dosis de $\mathrm{N}$ en los diferentes sistemas de rotación durante los dos ciclos de evaluación sugiere que la respuesta de los genotipos fue consistente para las diferentes dosis de N. Sin embargo, cuando se observó respuesta a dosis de N, el híbrido HB85 fue claramente superior en rendimiento a la variedad de polinización abierta B1 (Cuadro 6).

\section{CONCLUSIONES}

El efecto de rotación con abonos verdes y su relación con las dosis de fertilizante $\mathrm{N}$ confirma las posibilidades de substitución de las dos fuentes para la producción de maíz.

El factor principal que condicionó la respuesta a dosis de $\mathrm{N}$ bajo sistemas de abono verde fue la cantidad de $\mathrm{N}$ almacenado en la biomasa superficial de las leguminosas.

El tratamiento de semilla con insecticida fue esencial para lograr una densidad crítica de plantas que fue determinante para la expresión de respuesta a dosis de $\mathrm{N}$ en los diferentes sistemas de rotación evaluados. 
Cuando se observó respuesta a la aplicación de N, el híbrido HB85 fue superior en rendimiento a la variedad de polinización abierta B 1 .

\section{LITERATURA CITADA}

ARMUELLES BOUTET, R.A. 1992. Comercialización de fertilizantes y pesticidas en Centroamérica: el caso de los granos básicos. Colección: Temas de Seguridad Alimentaria No. 12. Programa de Seguridad Alimentaria CADESCA. Apdo. Aéreo 5215 Zona 5, Panamá. 131p

BOULDIN, D.R.; QUINTANA, J.; SUHET, A.. 1989. Evaluation of mineralization potential of 1egume residues. En: (Claude, N. ed) TropSoils Technical Report. 1986- 1987. North Carolina State University. Raleigh, N.C. pp 304-305.

NATIONAL ACADEMY OF SCIENCES. 1991. Toward sustainability: A plan for collaborative research on agriculture and natural resource management. National Academy Press. Washington D.C. p 66-90
SMYTH, T. J,; CRAVO, M. S.; MELGAR, R. J. 1991. Nitrogen supplied to corn by legumes in a central amazon oxisol. Trop. Agric. (Trinidad). 68: 366-372

WADE, M.K.; SANCHEZ, P.A. 1983. Mulching and green manure applications for continuous crop production in the amazon basin. Agron. J. 75: 39-45

YOST, R.S.; EVANS, D.O.; SAID, N.A.. 1985. Tropical legumes for $\mathrm{N}$ production: growth and $\mathrm{N}$ content in relation to soil pH. Trop. Agric. (Trinidad). 62: 20-24

ZEA, J.L.; BARRETO, H.J.; SAIN, G.E.; BOLAÑOS, J.; RAUN, W.R 1991. Efecto de intercalar leguminosas a diferentes dosis de fósforo sobre el rendimiento de maíz (Zea Mays L.) en 24 ensayos a través de Centroamérica. En: Análisis de los Ensayos Regionales de Agronomía, 1990. p 27-41. Programa Regional de Maíz para Centro América y El Caribe. 